\title{
miRNA-223-3p regulates NLRP3 to promote apoptosis and inhibit proliferation of hep3B cells
}

\author{
LINGFENG WAN $^{1}$, XIN YUAN ${ }^{2}$, MANTIAN LIU $^{1}$ and BOYU XUE ${ }^{1}$ \\ ${ }^{1}$ Department of Infectious Disease, Affiliated Hospital of Nanjing University of \\ Traditional Chinese Medicine; ${ }^{2}$ Department of Internal Medicine, The First Clinical Medical College, \\ Nanjing University of Chinese Medicine, Nanjing, Jiangsu 210029, P.R. China
}

Received March 16, 2017; Accepted August 18, 2017

DOI: $10.3892 /$ etm.2017.5667

\begin{abstract}
Hepatocellular carcinoma (HCC) is a major malignant tumor type with a high incidence and mortality. Infection with hepatitis virus is a high-risk factor. Previous studies have demonstrated that microRNA (miR)-223 was downregulated in HCC tissues. NOD-like receptor family, pyrin domain containing 3 (NLRP3)-is a potential target of miR-223 and has a vital role in hepatitis infection. The present study was performed to investigate the role of miR-223 in the proliferation and apoptosis of HCC cells through regulating NLRP3. A dual luciferase reporter assay was performed to confirm the direct interaction between miR-223-3p and the 3 ' untranslated region of NLRP3 mRNA. Hep3B cells were then transfected with miR-223 mimics and the proliferation and apoptosis were determined by an MTT and a flow cytometric assay, respectively. The expression of NLRP3 and caspase-1 was analyzed at the mRNA as well as at the protein level by reverse transcription-quantitative polymerase chain reaction and western blot analysis, respectively. The secretion of interleukin (IL)- $1 \beta$ and IL-18 in the culture supernatants was measured by ELISA. The dual luciferase assay confirmed NLRP3 as a direct target of miR-223. Overexpression of miR-223 in hep3B cells significantly suppressed cell proliferation and promoted apoptosis. Furthermore, the expression of NLRP3 was downregulated by miR-223 transfection. Certain downstream factors of the NLRP3 pathway were also downregulated following overexpression of miR-223. Caspase-1 was decreased at the transcriptional level and the cleaved caspase-1 was decreased at the protein level. Secretion of IL-1 $\beta$ and IL-18 into the culture medium by cells transfected with miR-223 was lower than that by the control cells. In conclusion, the tumor suppressor
\end{abstract}

Correspondence to: Dr Boyu Xue, Department of Infectious Disease, Affiliated Hospital of Nanjing University of Traditional Chinese Medicine, 155 Hanzhong Road, Nanjing, Jiangsu 210029, P.R. China

E-mail: xueboyu9502@hotmail.com

Key words: miR-223, hepatocellular carcinoma, NLRP3, proliferation, apoptosis role of miR-223 was associated with the regulation of NLRP3 inflammasome components. miR-223 inhibited HCC cell proliferation and promoted apoptosis by directly targeting NLRP3. Downstream production of caspase-1, IL-1 $\beta$ and IL-18 were also repressed by miR-223. These results provided insight into the association between the innate immune system and the genesis of HCC.

\section{Introduction}

Hepatocellular carcinoma (HCC) is a major malignant cancer type worldwide. It is estimated that $>748,000$ new cases are diagnosed each year, a majority of which are in resource-poor countries (1). China has a high disease burden of HCC, with 466,100 new HCC cases and 422,100 HCC-associated mortalities in 2015 (2). The progression of HCC is rapid and the prognosis is poor. Patients who develop HCC usually die within 12 months and the median survival time after HCC diagnosis is $<6$ months (3). The understanding of the molecular mechanisms of the pathogenesis of HCC still requires to be enhanced.

The most common risk factor for $\mathrm{HCC}$ is viral hepatitis infection, including infection with hepatitis B virus (HBV) and $\mathrm{HCV}$. Combined HBV and HCV infections are responsible for $85 \%$ of HCC cases (4). Activation of the NOD-like receptor family, pyrin domain containing 3 (NLRP3) inflammasome is commonly observed in the immune response to viral infections, including those with HBV and HCV (5-7). The NLRP3 inflammasome is a multiprotein complex, which is involved in caspase-1 activation. The major component of the NLRP3 inflammasome is NLRP3, a pyrin-containing protein that belongs to the nucleotide-binding oligomerization domain-like receptors. Caspase-1 induced by NLRP3 inflammasome activation is required for the maturation and secretion of the pro-inflammatory cytokines interleukin (IL)-18 and IL-1 $\beta$ (8). NLRP3 inflammasome activation by hepatitis infection finally results in hepatocyte pyroptosis, apoptosis and fibrosis $(9,10)$.

Post-transcriptional regulation by microRNAs (miRNAs/miRs) is an important mechanism to control gene expression. miRNAs have regulatory functions through binding to complementary sequences in the 3'-untranslated region (3'-UTR) of their target mRNAs via base pairing to degrade them or inhibit their translation (11). As indicated 
in a study by Guo et al (12), decreases in protein production in mammalian cells were mainly due to regulatory interactions with ectopic and endogenous miRNA. In murine macrophages, NLRP3 transcription is tightly regulated by miR-223 through an evolutionarily conserved binding interaction between the 3'-UTR of NLRP3 mRNA and miR-223-3p, indicating that NLRP3 expression is also regulated by miRNAs (13). miR-223 is a negative regulator of inflammation and usually upregulated in cells of myeloid lineages (14). miR-223 is also associated with HCC development and commonly downregulated in HCC tissues (15). Based on the abovementioned previous studies, including those reporting that chronic hepatitis infection is associated with NLRP3 activation as major cause of hepatic damage and tumorigenesis, it was deduced that NLRP3 has a role in HCC development and is regulated by miR-223. Therefore, the aim of the present study was to assess the influence of NLRP3 on pathological features of HCC cells and to verify its potential regulation by miR-223.

\section{Materials and methods}

Cell line and culture. The Hep 3B2.1-7 cell line was purchased from the American Type Culture Collection (Manassas, VA, USA) and used in all of the experiments. The cell line was derived from a chronic HBV carrier and suitable for transfection. The cells were cultured as recommended with Dulbecco's modified Eagle's medium (DMEM; Gibco ${ }^{\circledR}$; Thermo Fisher Scientific, Inc., Waltham, MA, USA) supplemented with $10 \%$ fetal bovine serum (FBS; Gibco ${ }^{\circledR}$; Thermo Fisher Scientific, Inc.) in a humidified atmosphere of $95 \%$ air and $5 \% \mathrm{CO}_{2}$ at $37^{\circ} \mathrm{C}$.

Cell transfection. Hep3B cells were transfected with mature miR-223 mimics (Biomics Biopharma, Nantong, China; 5'-UGUCAGUUUGUCAAAUACCCC-3') to investigate the regulatory role of $\mathrm{miR}-223$. All of the transfections were performed using the Lipofectamine 3000 reagent (Invitrogen ${ }^{\circledR}$; Thermo Fisher Scientific, Inc.) according to the manufacturer's instructions. Non-homologous miRNA mimics control was used as a negative control (NC). Cells were trypsinized and collected at $24 \mathrm{~h}$ after transfection for cell proliferation and apoptosis assays. miR-223 mimics and miRNA mimics control were purchased from RiboBio Co., Ltd. (Guangzhou, China).

Dual luciferase reporter assay. The predicted miR-223 binding site of the NLRP3 3'-UTR sequence (5'-CGCUAUCUUUCU AUUAACUGACCAUAA-3') or mutant sequence(5'-CGC UAUCUUUCUAUUAUGACUCCAUAA-3') was cloned into the pMIR-REPORT vector $\left(\right.$ Ambion $^{\circledR}$; Thermo Fisher Scientific, Inc.) to construct the NLRP3-3'UTR-WT plasmid or the NLRP3-3'UTR-Mut plasmid. HEK293T cells were co-transfected with 40 ng plasmid containing NLRP3 3'-UTR or mutant sites, $40 \mathrm{ng}$ pRL-TK Renilla luciferase reporter plasmid (Ambion ${ }^{\circledR}$, Thermo Fisher Scientific, Austin, TX, USA) and $20 \mathrm{ng}$ miR-223 mimics. Renilla luciferase activity was measured $48 \mathrm{~h}$ after transfection by using the dual luciferase reporter assay system (Promega Corp., Madison, WI, USA).
Cell proliferation assay and apoptosis assay. Cell proliferation was determined by the MTT method. Cells were seeded in a 96-well plate $(3,000$ cells/well) with $200 \mu \mathrm{l}$ medium and cultured for $48 \mathrm{~h}$. For the proliferation assay, $20 \mu \mathrm{l} \mathrm{MTT}$ solution $(5 \mathrm{mg} / \mathrm{ml})$ was added to each well, followed by incubation for $2 \mathrm{~h}$. The cell density was calculated according to the optical density at $450 \mathrm{~nm}$ determined using a microplate reader. For the apoptosis assay, collected cells were stained with Annexin V-fluorescein isothiocyanate (FITC) and propidium iodide solution (included in the Annexin V-FITC Apoptosis Detection kit (cat no. C1062; Beyotime Institute of Biotechnology, Haimen, China) and incubated in the dark for $20 \mathrm{~min}$. The apoptotic rate was then measured by flow cytometry. For each sample, signals of $10^{5}$ cells were detected.

RNA isolation and $m R N A$ detection. Total RNA was extracted from harvested cells by using the RNAiso Plus kit (cat no. 9180; Takara Biotechnology, Dalian, China). First-strand complementary (c)DNA synthesis was performed by using the PrimeScript 1st Strand cDNA Synthesis kit (cat no. 6110A; Takara Biotechnology). To determine the gene expression levels, quantitative polymerase chain reaction (qPCR) was performed using the QuantiTect SYBR Green PCR kit (cat no. 204145; Qiagen, Hilden, Germany). The primers used for reverse transcription and $\mathrm{qPCR}$ are summarized in Table I. U6 small nuclear RNA was used as an internal control for miR-223. $\beta$-actin was used as an internal control for the other genes. The relative mRNA levels of the target genes were normalized to $\beta$-actin by using the $2^{-\Delta \Delta \mathrm{Cq}}$ method (16).

Western blot analysis. Total cell lysates were prepared in SDS lysis buffer (Beyotime Institute of Biotechnology). The protein concentration was quantified by using the BCA Protein Assay kit (Beyotime Institute of Biotechnology). Protein lysates (30 $\mu \mathrm{g} /$ lane) were separated by $10 \%$ SDS-PAGE and electro-transferred onto a nitrocellulose membrane (EMD Millipore, Billerica, MA, USA). The membrane was blocked with $5 \%$ skimmed milk and then incubated overnight at $4{ }^{\circ} \mathrm{C}$ with the following primary antibodies: Rabbit polyclonal antibody to NLRP3 (1:2,000 dilution; cat. no. ab98151), rabbit polyclonal antibody to caspase-1 (1:2,000 dilution; cat. no. ab1872) and rabbit polyclonal antibody to $\beta$-actin $(1: 2,000$ dilution; cat. no. ab8227). Blots were then incubated with horseradish peroxidase-conjugated goat anti-rabbit immunoglobulin G (1:3,000 dilution; cat. no. ab205718) at room temperature for $1 \mathrm{~h}$. The protein bands were visualized by using the BeyoECL Plus kit (cat no. P0018; Beyotime Institute of Biotechnology). All of the antibodies were purchased from Abcam Inc. (Cambridge, MA, USA). $\beta$-actin was used as an internal control.

Cytokine detection. The inflammatory cytokines IL-1 $\beta$ and IL-18 were detected in the cell culture supernatants. The Human IL-1 Beta PicoKine ELISA kit (cat no. EK0392) and the Human IL-18 PicoKine ELISA kit (cat no. EK0864; Boster Biological Technology, Wuhan, China) were used to measure the concentrations of the cytokines.

Statistical analysis. All data were analyzed by using SPSS software version 19.0 (IBM Corp., Armonk, NY, USA). All 

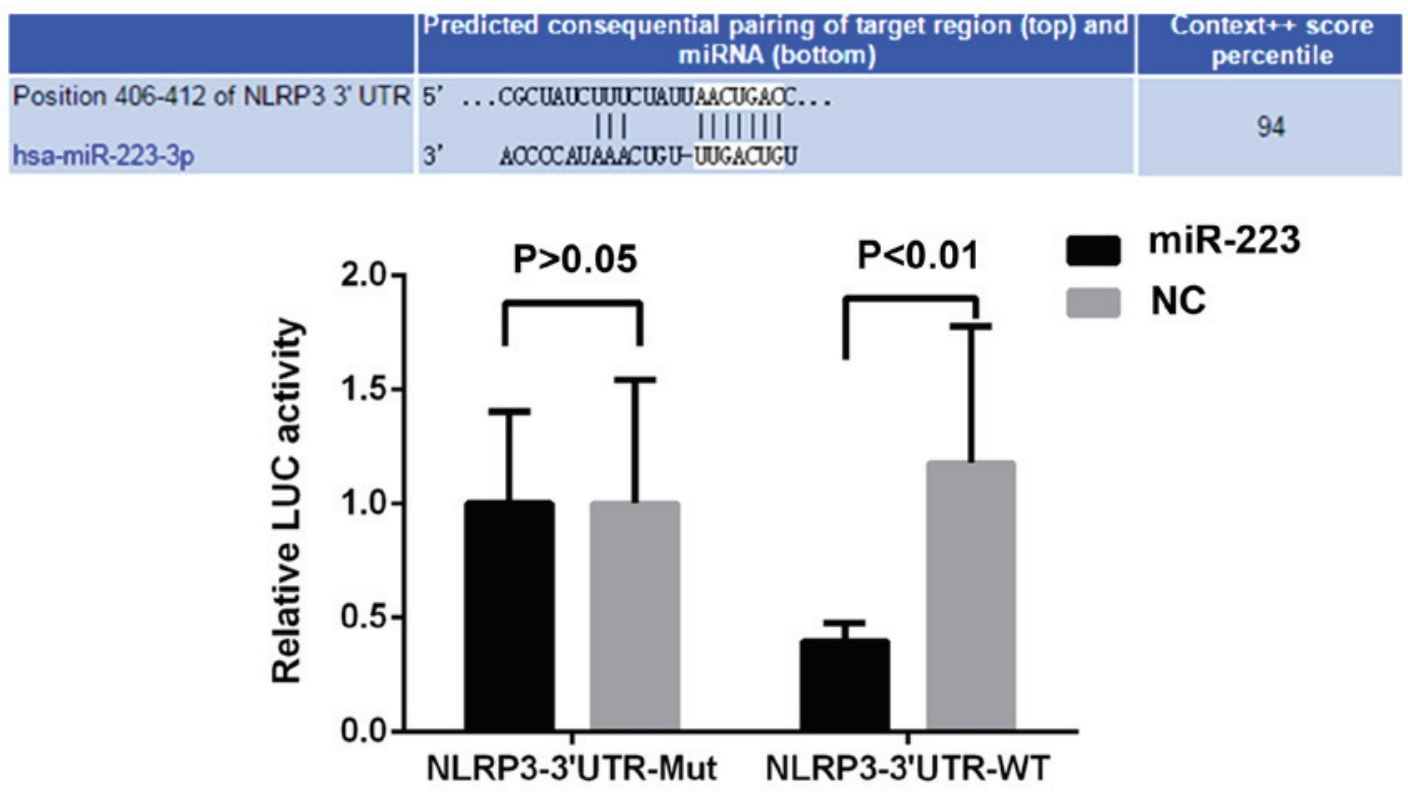

Figure 1. miR-223 regulates NLRP3 expression by directly targeting the 3'-UTR of its mRNA. Predicted potential binding sites of miR-223 and NLRP3 mRNA are displayed in the upper panel. A dual luciferase reporter assay was performed. Luciferase activities of plasmids with WT or Mut sequences were assessed in HEK293T cells co-transfected with miR-223 mimics or non-homologous NC. NLRP3-3'UTR-WT, luciferase reporter plasmid containing the WT 3'UTR sequence of NLRP3; BL, blank control; UTR, untranslated region; miR, microRNA; WT, wild-type; Mut, mutant; NLRP3, NACHT, LRR and PYD domains-containing protein 3; hsa, Homo sapiens; NC, negative control; LUC, luciferase.

experiments were repeated at least 3 times and representative images of one experiment are displayed. Values are expressed as the mean \pm standard deviation (SD). Student's t-test was performed to analyze differences between two groups. One-way analysis of variance was used to compare between multiple groups followed by a Newman-Keuls multiple comparison test. $\mathrm{P}<0.05$ was considered to indicate a statistically significant difference.

\section{Results}

miR-223 interacts with the 3'-UTR of NLRP3 mRNA. Bases 406-412 of the 3'-UTR of NLRP3 mRNA were predicted to be a binding site for miR-223-3p using TargetScanHuman (http://www.targetscan.org/vert_71/) (Fig. 1A). To confirm the presence of a direct interaction between the two molecules, a dual-luciferase reporter assay was performed. As presented in Fig. 1B, co-transfection with miR-223 reduced the luciferase activity of the plasmid containing the wild-type of the respective fragment of NLRP3 3'-UTR. However, the luciferase activity of the plasmid containing the mutant NLRP3 3'-UTR fragment was not affected by co-transfection with miR-223 mimics or negative control. These results indicated that miR-223 directly interacted with the 3'-UTR of NLRP3 mRNA.

miR-223 influences the proliferation and apoptosis of hep $3 B$ cells. Next, the effect of miR-223 overexpression on the proliferation or apoptosis of hep3B cells was assessed. When hep3B cells were transfected with miR-223, a significant decrease of cell proliferation was detected by using the MMT method (Fig. 2A). The replication rate of the cells transfected with miR-223 declined to $\sim 65 \%$ of that of the cells transfected with mimics control $(\mathrm{P}<0.05)$. At the same time, miR-223 increased the apoptotic rate (Fig. 2B). The apoptotic rate increased to $27.1 \%$ following transfection with miR-223, which was almost 4 -fold of the rate in the blank control group or the negative control group $(\mathrm{P}<0.01)$. These results indicated that miR-223 has a tumor suppressor role in hep3B cells.

miR-223 regulates the expression of NLRP3 inflammasome components and downstream cytokines. As the 3'-UTR of NLRP3 mRNA was demonstrated to contain a direct target region of miR-223, it was further assessed whether the miR-223 levels correlated with the expression of the NLRP3 inflammasome regulation pathway. As presented in Fig. 3A and B, NLRP3 expression in hep3B cells was inhibited at the mRNA as well as the protein level following transfection with miR-223. The NLRP3 inflammasome contains a caspase-recruitment domain. Upon activation of the NLRP3 inflammasome, pro-caspase-1 is recruited to the inflammasome complex and cleaved to generate caspase-1. Consistent with the decrease of NLRP3 expression, caspase-1 expression was also downregulated at the mRNA and protein level following overexpression of miR-223, while the expression of pro-caspase-1 remained unchanged (Fig. 3A-C). Activation of caspase- 1 subsequently cleaves pro-IL- $1 \beta$ and pro-IL-18 into mature IL-1 $\beta$ and IL-18, so the secretion of these two cytokines was also measured. The concentration of IL-1 $\beta$ and IL-18 in the miR-223 transfection group was lower than that in the mimics and the blank control group (Fig. 3D). The results indicated that miR-223 suppressed NLRP3 expression and subsequently reduced the levels of caspase-1, IL-1 $\beta$ and IL-18.

\section{Discussion}

The role of the NLRP3 inflammasome in cancer remains controversial. The present study observed that downregulation 

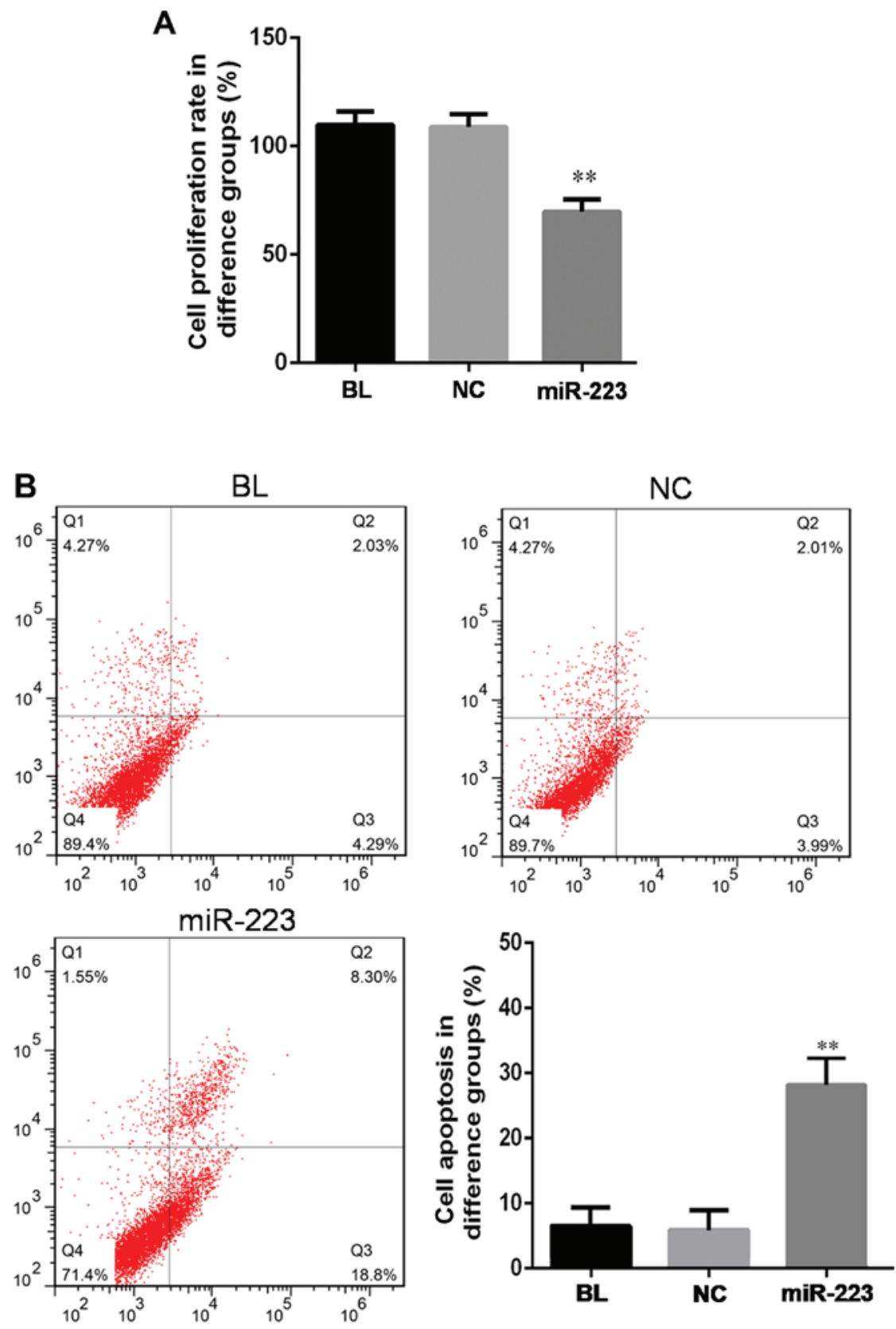

Figure 2. Overexpression of miR-223 inhibits the proliferation and promotes apoptosis of hep3B cells. (A) Cell proliferation determined using the MTT method. (B) Apoptosis was measured by flow cytometry. ${ }^{* *} \mathrm{P}<0.01$ vs. BL group. BL, blank control; NC, non-homologous negative control; miR, microRNA.

of NLRP3, caspase-1, IL-1 $\beta$ and IL-18 favored apoptosis of the HCC cell line hep3B, indicating that activation of the NLRP3 inflammasome pathway has a procarcinogenic effect. This type of role was also reported in colitis-associated cancer (17). During chemotherapy, the NLRP3 inflammasome in dendritic cells was reported to be activated to induce IL-1 $\beta$-dependent adaptive immunity against thymoma (18). The NLRP3 inflammasome was also revealed to be activated by chemotherapy-triggered cathepsin B release in myeloid-derived suppressor cells and blunted the anticancer efficacy of the chemotherapy by IL-1 $\beta$ induction (19). However, other studies also reported a protective role of the NLRP3 inflammasome in cancer. The NLRP3 inflammasome activated the tumoricidal function of natural killing cells and suppressed colorectal cancer metastasis in the liver (20). A study by Wei et al (21) indicated that expression of NLRP3 inflammasome components was upregulated in hepatic parenchymal cells with hepatitis infection or cirrhosis but downregulated in HCC tissues when compared with adjacent normal tissues. A further study by the same group indicated that treatment with $17 \beta$-estradiol inhibited the invasion and migration of HCC cells through estrogen/mitogen-activated protein kinase-mediated upregulation of the NLRP3 inflammasome (22). These two studies revealed a dynamic expression pattern of NLRP3 during multiple stages of HCC progression; however, the authors did not perform a stratified analysis by the hepatitis infection status in HCC patients. NLRP3 expression may also be cell linage-associated. The HCC cell lines used by Wei et al $(21,22)$ are all non-B or non-C associated (BEL7402, SMMC7721 and HepG2), while the hep3B cell 
A

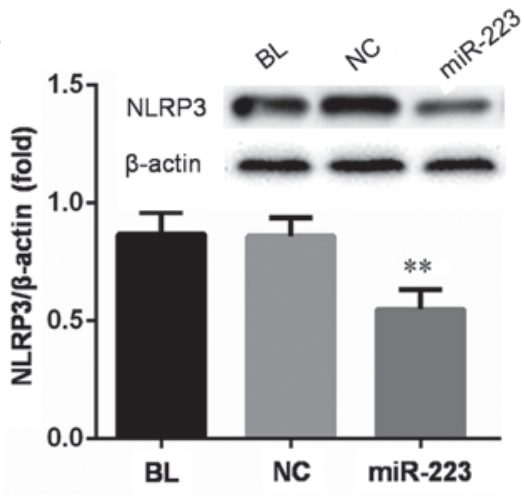

C
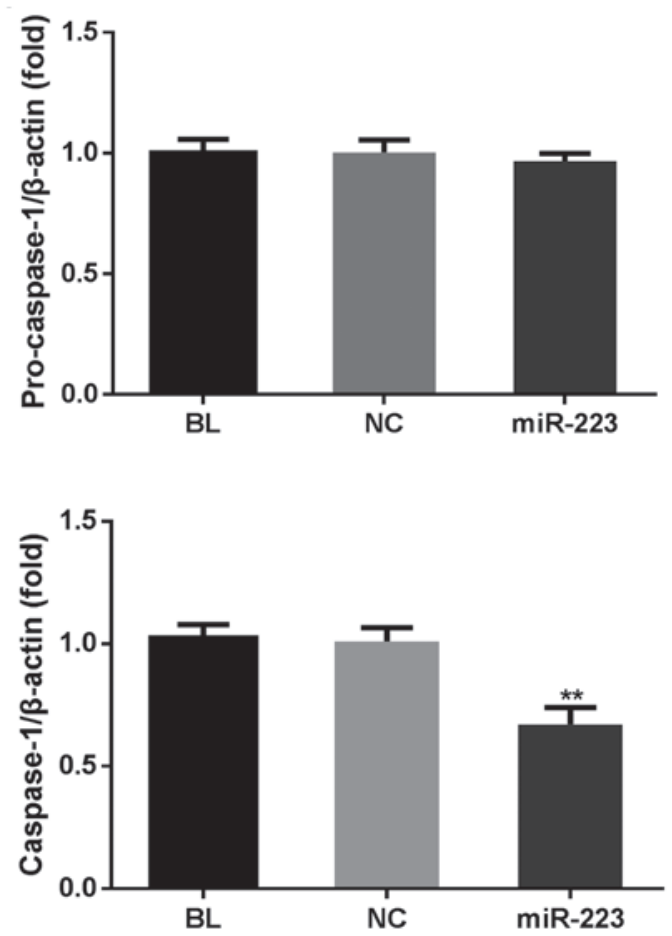

B

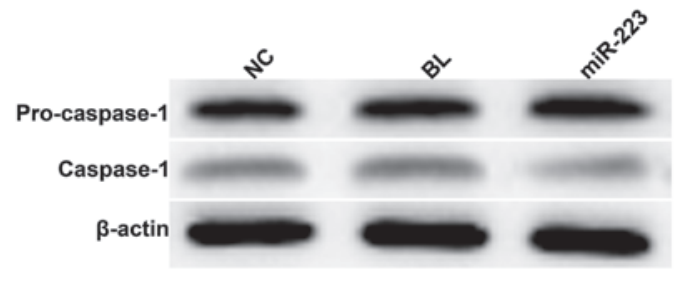

D
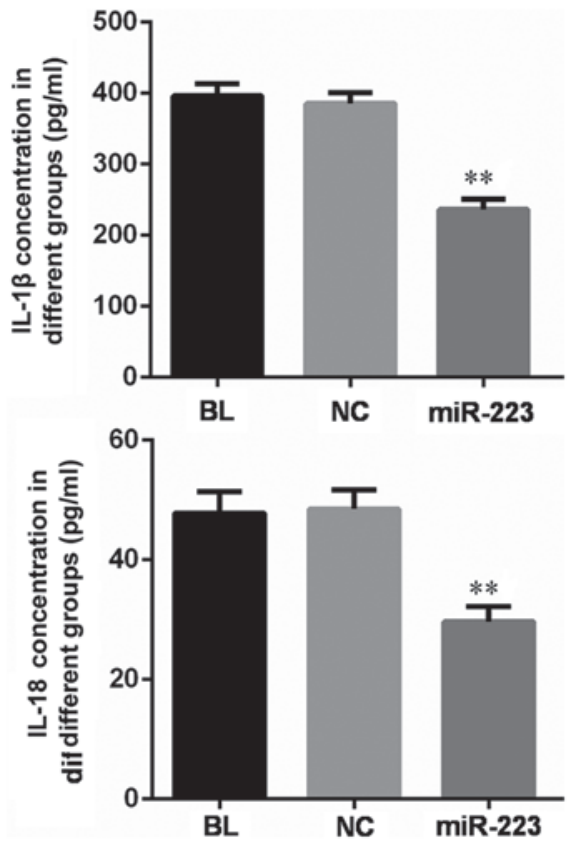

Figure 3. Transfection of miR-223 mimics leads to downregulation of NLRP3 inflammasome components. (A) Relative mRNA levels of NLRP3 and caspase-1 normalized to $\beta$-actin and western blot image. (B) Western blot analysis of pro-caspase-1 and caspase-1. (C) Expression level of pro-caspase-1 and caspase-1 normalized to $\beta$-actin. (D) Concentrations of IL-1 $\beta$ and IL-18 in culture supernatants. ${ }^{* *} \mathrm{P}<0.01$ vs. BL group. BL, blank control; NC, non-homologous negative control; miR, microRNA; NLRP3, NACHT, LRR and PYD domains-containing protein 3; IL, interleukin.

line used in the present study is derived from an HBV-positive patient. The role of the NLRP3 inflammasome in HCC or other human cancers has yet to be elucidated by further studies. Furthermore, previous research has demonstrated that NF-kB may induce the inhibition of apoptosis-associated gene expression through TRAF1 (TNFR-associated factor 1), TRAF2, c-IAP1 (Inhibitor-of-apoptosis) and c-IAP2, which results in the suppression of caspase-1 expression in the inflammatory environment (23).

The results of the present study demonstrated that miR-223 promoted apoptosis and inhibited the proliferation of HCC cells by directly regulating NLRP3. The regulation of NLRP3 by miR-223 was also reported in other diseases. miR-223 was revealed to downregulate NLRP3 to inhibit inflammation, reduce brain edema and improve neurological functions after intracerebral hemorrhage (24). During Epstein-Barr virus (EBV) infection, decreased miR-223 expression led to overexpression of NLRP3 and IL-1 $\beta$-associated inflammation. EBV
miR-BART15 was reported to target the miR-223 binding site in the 3'-UTR of NLRP3 mRNA and thereby inhibited NLRP3 expression (25).

In addition to NLRP3, miR-223 has other targets in various solid tumor types. In HCC, downregulation of miR-223 was also correlated with an upregulation of stathmin1 (15). Insulin-like growth factor-1 receptor (IGF-1R) and cyclin-dependent kinase 2 were identified as direct targets of miR-223 in non-small cell lung cancer (26). Suppression of IGF-1R by miR-223 was also observed in HeLa cells and the osteosarcoma cell line MG63 (27,28). In MG63 cells, heat-shock protein 90B1 was another target of miR-223 (28). Artemin was reported to be a target of miR-223 in human esophageal carcinoma (29). miR-223 was also revealed to promote the invasion and metastasis of gastric cancer by regulating erythrocyte membrane protein band 4.1 like 3 (30). miR-223 also regulates transcription factor forkhead box $\mathrm{O} 1$ in multiple cancer cell lines, including the colorectal cancer 
Table I. Primers used for RT-qPCR.

A, Primers used for RT

\begin{tabular}{ll}
\hline RNA & \multicolumn{1}{c}{ Primer sequence } \\
\hline miR-223 & 5'-GTCGTATCCAGTGCAGGGTCCGAGGT \\
& ATTCGCACTGGATACGACTGGGGT-3' \\
U6 & 5'-GTCGTATCCAGTGCAGGGTCCGAGGT \\
& ATTCGCACTGGATACGACAAAATATGGA \\
& AC-3' \\
\hline
\end{tabular}

B, Primers used for $\mathrm{qPCR}$

\section{Gene/}

direction

Primer sequence

$\begin{array}{ll}\text { U6 } & \\ \text { Forward } & \text { 5'-CTCGCTTCGGCAGCACA-3' } \\ \text { Reverse } & \text { 5'-AACGCTTCACGAATTTGCGT-3' } \\ \text { miR-223 } & \\ \text { Forward } & \text { 5'-GTGCAGGGTCCGAGGT-3' } \\ \text { Reverse } & \text { 5'-CGGGCTGTCAGTTTGTCA-3' } \\ \text { NLRP3 } & \\ \text { Forward } & \text { 5'-GCAGCAAACTGGAAAGGAAG-3' } \\ \text { Reverse } & \text { 5'-CTTCTCTGATGAGGCCCAAG-3' } \\ \text { Caspase-1 } & \\ \text { Forward } & \text { 5'-CCGAAGGTGATCATCATCCA-3' } \\ \text { Reverse } & \text { 5'-ATAGCATCATCCTCAAACTCTTCTG-3' } \\ \begin{array}{l}\text { 3-actin } \\ \text { Forward }\end{array} & \text { 5'-AGGGGCCGGACTCGTCATACT-3' } \\ \text { Reverse } & \text { 5'-GGCGGCACCACCATGTACCCT-3' }\end{array}$

RT-qPCR, reverse transcription-quantitative polymerase chain reaction; miR, microRNA; NLRP3, NACHT, LRR and PYD domains-containing protein 3.

cell line HCT116, the cervical cancer cell line HeLa and the hepatoma cell HuH-7 (31). These studies comprehensively indicated multiple roles of miR-223 during tumorigenesis.

In conclusion, the present study demonstrated a regulatory effect of miR-223 on NLRP3 inflammasome components in HCC cells. These results provided insight into the association between the innate immune system and the genesis of HCC. Of note, the present study had certain limitations. Only one cell line, HCC, was used in all of the experiments. Considering the different biological features of HCC cell lines, replication of the experiments is required to fully reveal the link between the NLRP3 inflammasome and HCC development. Furthermore, in vivo tests are considered for further studies to confirm the in vitro results of the present study.

\section{Acknowledgements}

The study was financially supported by the 'Six Talent Projects' of Jiangsu Province (grant no. 2015-WSN-047), a Research
Project of the Affiliated Hospital of Nanjing University of Traditional Chinese Medicine (grant no. Y16019) and the Priority Academic Program Development of Jiangsu Higher Education Institutions.

\section{References}

1. Jemal A, Bray F, Center MM, Ferlay J, Ward E and Forman D: Global cancer statistics. CA Cancer J Clin 61: 69-90, 2011.

2. Chen W, Zheng R, Baade PD, Zhang S, Zeng H, Bray F, Jemal A, Yu XQ and He J: Cancer statistics in China, 2015. CA Cancer J Clin 66: 115-132, 2016.

3. Kew MC: Hepatocellular carcinoma: Epidemiology and risk factors. J Hepatocell Carcinoma 1: 115-125, 2014.

4. Perz JF, Armstrong GL, Farrington LA, Hutin YJ and Bell BP: The contributions of hepatitis B virus and hepatitis $C$ virus infections to cirrhosis and primary liver cancer worldwide. J Hepatol 45: 529-538, 2006.

5. Kanneganti T: Central roles of NLRs and inflammasomes in viral infection. Nat Rev Immunol 10: 688-698, 2010.

6. Chen W, Xu Y, Li H, Tao W, Xiang Y, Huang B, Niu J, Zhong J and Meng G: HCV genomic RNA activates the NLRP3 inflammasome in human myeloid cells. PLoS One 9: e84953, 2014.

7. Szabo G and Petrasek J: Inflammasome activation and function in liver disease. Nat Rev Gastro Hepat 12: 387-400, 2015.

8. Franchi L, Eigenbrod T, Muñoz-Planillo R and Nuñez G: The inflammasome: A caspase-1-activation platform that regulates immune responses and disease pathogenesis. Nat Immunol 10: 241-247, 2009.

9. Wree A, Eguchi A, Mcgeough MD, Pena CA, Johnson CD, Canbay A, Hoffman HM and Feldstein AE: NLRP3 inflammasome activation results in hepatocyte pyroptosis, liver inflammation, and fibrosis in mice. Hepatology 59: 898-910, 2014.

10. Aachoui Y, Sagulenko V, Miao EA and Stacey KJ: Inflammasome-mediated pyroptotic and apoptotic cell death, and defense against infection. Curr Opin Microbiol 16: 319-326, 2013.

11. Bartel DP: MicroRNAs: Target recognition and regulatory functions. Cell 136: 215-233, 2009.

12. Guo H, Ingolia NT, Weissman JS and Bartel DP: Mammalian microRNAs predominantly act to decrease target mRNA levels. Nature 466: 835-840, 2010.

13. Bauernfeind F, Rieger A, Schildberg FA, Knolle PA, Schmid-Burgk JL and Hornung V: NLRP3 inflammasome activity is negatively controlled by miR-223. J Immunol 189: 4175-4181, 2012.

14. Ramkissoon SH, Mainwaring LA, Ogasawara Y, Keyvanfar K, McCoy JP Jr, Sloand EM, Kajigaya S and Young NS: Hematopoietic-specific microRNA expression in human cells. Leuk Res 30: 643-647, 2006.

15. Wong QW, Lung RW, Law PT, Lai PB, Chan KY, To KF and Wong N: MicroRNA-223 is commonly repressed in hepatocellular carcinoma and potentiatesexpression of stathmin1. Gastroenterology 135: 257-269, 2008.

16. Livak KJ and Schmittgen TD: Analysis of relative gene expression data using real-time quantitative PCR and the 2(-Delta Delta C(T)) method. Methods 25: 402-408, 2001.

17. Allen IC, Tekippe EM, Woodford RM, Uronis JM, Holl EK, Rogers AB, Herfarth HH, Jobin C and Ting JP: The NLRP3 inflammasome functions as a negative regulator of tumorigenesis during colitis-associated cancer. J Exp Med 207: 1045-1056, 2010.

18. Ghiringhelli F, Apetoh L, Tesniere A, Aymeric L, Ma Y, Ortiz C, Vermaelen K, Panaretakis T, Mignot G, Ullrich E, et al: Activation of the NLRP3 inflammasome in dendritic cells induces IL-1beta-dependent adaptive immunity against tumors. Nat Med 15: 1170-1178, 2009.

19. Bruchard M, Mignot G, Derangère V, Chalmin F, Chevriaux A, Végran F, Boireau W, Simon B, Ryffel B, Connat JL, et al: Chemotherapy-triggered cathepsin B release in myeloid-derived suppressor cells activates the Nlrp3 inflammasome and promotes tumor growth. Nat Med 19: 57-64, 2013.

20. Dupaul-Chicoine J, Arabzadeh A, Dagenais M, Douglas T, Champagne C, Morizot A, Rodrigue-Gervais IG, Breton V, Colpitts SL, Beauchemin N and Saleh M: The Nlrp3 inflammasome suppresses colorectal cancer metastatic growth in the liver by promoting natural killer cell tumoricidal activity. Immunity 43: 751-763, 2015. 
21. Wei Q, Mu K, Li T, Zhang Y, Yang Z, Jia X, Zhao W, Huai W, Guo P and Han L: Deregulation of the NLRP3 inflammasome in hepatic parenchymal cells during liver cancer progression. Lab Invest 94: 52-62, 2014.

22. Wei Q, Guo P, Mu K, Zhang Y, Zhao W, Huai W, Qiu Y, Li T, Ma X, Liu Y, et al: Estrogen suppresses hepatocellular carcinoma cells through ER $\beta$-mediated upregulation of the NLRP3 inflammasome. Lab Invest 95: 804-816, 2015.

23. Chen K, Mi MT and Yu XP: Effects of taurine on apoptosis of photoreceptors and NF-kB/Caspase-1 pathway in photochemical damage. Acta Nutrimenta Sinica 8: 296-299, 2006 (In Chinese).

24. Yang N, Ekanem NR, Sakyi CA and Ray SD: Hepatocellular carcinoma and microRNA: New perspectives on therapeutics and diagnostics. Adv Drug Deliv Rev 81: 62-74, 2015.

25. Haneklaus M, Gerlic M, Kurowska Stolarska M, Rainey AA, Pich D, Mcinnes IB, Hammerschmidt W, Neill LA and Masters SL: Cutting edge: miR-223 and EBV miR-BART15 regulate the NLRP3 inflammasome and IL-1 $\beta$ production. J Immunol 189: 3795-3799, 2012.

26. Nian W, Ao X, Wu Y, Huang Y, Shao J, Wang Y, Chen Z, Chen F and Wang D: miR-223 functions as a potent tumor suppressor of the Lewis lung carcinoma cell line by targeting insulin-like growth factor-1 receptor and cyclin-dependent kinase 2. Oncol Lett 6: 359-366, 2013.

27. Jia CY, Li HH, Zhu XC, Dong YW, Fu D, Zhao QL, Wu W and Wu XZ: MiR-223 suppresses cell proliferation by targeting IGF-1R. PLoS One 6: e27008, 2011.
28. Li G, Cai M, Fu D, Chen K, Sun M, Cai Z and Cheng B: Heat shock protein $90 \mathrm{~B} 1$ plays an oncogenic role and is a target of microRNA-223 in human osteosarcoma. Cell Physiol Bioche 30: 1481-1490, 2012.

29. Li S, Li Z, Guo F, Qin X, Liu B, Lei Z, Song Z, Sun L, Zhang HT, You $\mathrm{J}$ and Zhou Q: miR-223 regulates migration and invasion by targeting Artemin in human esophageal carcinoma. J Biomed Sci 18: 24, 2011.

30. Li X, Zhang Y, Zhang H, Liu X, Gong T, Li M, Sun L, Ji G, Shi Y, Han Z, et al: miRNA-223 promotes gastric cancer invasion and metastasis by targeting tumor suppressor EPB41L3. Mol Cancer Res 9: 824-833, 2011.

31. Wu L, Li H, Jia CY, Cheng W, Yu M, Peng M, Zhu Y, Zhao Q, Dong YW, Shao K, et al: MicroRNA-223 regulates FOXO1 expression and cell proliferation. FEBS Lett 586: 1038-1043, 2012.

This work is licensed under a Creative Commons Attribution 4.0 International (CC BY 4.0) License. 